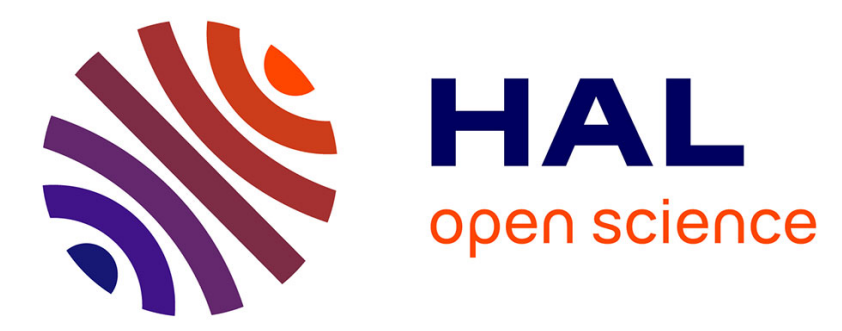

\title{
Stability guaranteed active fault tolerant control of networked control systems
}

Shanbin Li, Dominique Sauter, Christophe Aubrun, Joseph Julien Yamé

\section{To cite this version:}

Shanbin Li, Dominique Sauter, Christophe Aubrun, Joseph Julien Yamé. Stability guaranteed active fault tolerant control of networked control systems. European Control Conference, ECC'07, Jul 2007, Kos, Greece. pp.CDROM. hal-00162461

\section{HAL Id: hal-00162461 https://hal.science/hal-00162461}

Submitted on 13 Jul 2007

HAL is a multi-disciplinary open access archive for the deposit and dissemination of scientific research documents, whether they are published or not. The documents may come from teaching and research institutions in France or abroad, or from public or private research centers.
L'archive ouverte pluridisciplinaire HAL, est destinée au dépôt et à la diffusion de documents scientifiques de niveau recherche, publiés ou non, émanant des établissements d'enseignement et de recherche français ou étrangers, des laboratoires publics ou privés. 


\title{
Stability Guaranteed Active Fault Tolerant Control of Networked Control Systems
}

\author{
Shanbin Li, Dominique Sauter, Christophe Aubrun, Joseph Yamé
}

\begin{abstract}
The stability guaranteed active fault tolerant control against actuators failures in networked control systems (NCS) is addressed. A detailed design procedure is formulated as a convex optimization problem which can be efficiently solved by existing software. An illustrative example is given to show the efficiency of the proposed method for NCS.
\end{abstract}

\section{INTRODUCTION}

Fault tolerant control (FTC) techniques against actuator faults can be classified into two groups: passive and active approaches. In passive FTC systems, a single controller with fixed structure/parameters is used to deal with all possible failure scenarios which are assumed to be known a priori. Consequently, the passive controller is usually conservative. Furthermore, if a failure out of those considered in the design occurs, the stability and performance of the closedloop system might not be guaranteed. Such potential limitations of passive approaches provide a strong motivation for the development of methods and strategies for active FTC (AFTC) systems.

In contrast to passive FTC systems, AFTC techniques rely on a real-time fault detection and isolation (FDI) scheme and a controller reconfiguration mechanism. Such techniques allow a flexibility to select different controllers according to different component failures, and therefore better performance of the closed-loop system can be expected. However, this holds true when the FDI process does not make an incorrect or delayed decision [1]. Some preliminary results have been obtained on AFTC which is immune to imperfect FDI process [2], [3]. In reference [4], the latter issue is further discussed in a classical setting (i.e., point-to-point control) by using the guaranteed cost control approach and online controller switching in order to ensure stability of the closedloop system at all times. The aim of this paper is to extend the results in reference [4] to plants controlled over digital communication networks. In such networks, the information transfer from sensors to controllers and from controllers to actuators is not instantaneous but suffers communication delays. These network-induced delays may impact adversely on the stability and performance of the control system [5], [6]. Networked control systems (NCS) are now pervasive and such systems are long-running real-time systems which should function in a correct manner even in the presence of

This work is supported by the French National Research Agency (ANR) project SafeNECS under grant No. ANR-ARA SSIA-NV-15

The authors are with the Centre de Recherche en Automatique de Nancy (CRAN-UMR 7039), Nancy-Université, CNRS, BP239, F-54506 Vandoeuvre-lès-Nancy Cedex , France

Correspondence to: Joseph Yamé (or Dominique Sauter) Email: Joseph.Yame(Dominique.Sauter)@cran.uhp-nancy.fr failures. This makes the issue of fault tolerant control in NCS an important one and entails designing strategies to cope with some of the fundamental problems introduced by the network such as bandwidth limitations, quantization and sampling effects, message scheduling and communication delays. Motivated by the above considerations, we address the problem of fault tolerant control in NCS with time-varying delays. Specifically, we extend the results of reference [4] for the stabilization of a plant, subject to model uncertainties and actuator faults, which is controlled over a communication network that induces time-varying but bounded delays.

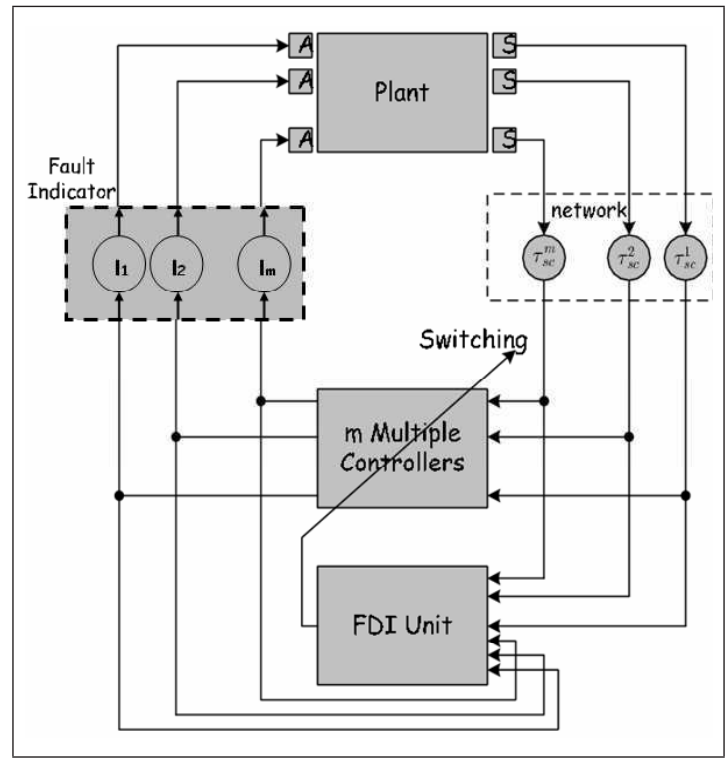

Fig. 1. Networked control system with actuator failures

\section{Problem Statement}

Figure 1 shows the basic networked control architecture considered in this paper and which consists of a single uncertain plant, with few sensors and actuators, controlled by a digital controller in a centralized structure. The delays induced by the network in the closed-loop control system are modelled as time-varying quantities $\tau(k)=\tau_{k}^{s c}$ arising from the communication delays between sensors and controllers at time $k$. For the simplicity of analysis, we assume that the controllers and actuators are located at the same side and there are no transmission time-delays between the controllers and actuators. The actuators might be subject to faults. Thus, taking into account the potential failures of actuators, the interconnection of the uncertain discrete-time plant and a discrete-time controller through the digital communication 
link as depicted in figure 1 can be described by the following dynamical and state-delayed feedback equations:

$$
\begin{aligned}
x(k+1) & =(A+D \Delta(k) E) x(k)+B \mathcal{L} u(k), \\
x(0) & =x_{0}, \\
u(k) & =K x(k-\tau(k))
\end{aligned}
$$

where $x(k) \in \mathcal{R}^{n}$ is the state of the uncertain plant, $u(k) \in \mathcal{R}^{m}$ is the control input, $A, B, D, E$ are all real constant matrices. Matrix $K$ is the controller gain matrix to be designed and $\Delta(k)$ is an uncertain time-varying matrix satisfying the bound $\Delta(k)^{T} \Delta(k) \leq I$ where $I$ denotes the identity matrix with appropriate dimension. The fault indicator matrix $\mathcal{L}$ is given by

$$
\mathcal{L}=\operatorname{diag}\left\{l_{1}, \ldots, l_{m}\right\}
$$

with $l_{j} \in\{0,1\}$ for $j=1,2, \ldots, m$ and where $l_{j}=1$ means that the $j$ th actuator is in healthy state, whereas the $j$ th actuator is meant to experience a total failure when $l_{j}=0$. Having a finite number of actuators, the set of possible related failure modes is also finite and, by abuse of notation, we denote this set by

$$
\mathcal{L}=\left\{\mathcal{L}^{1}, \mathcal{L}^{2}, \ldots, \mathcal{L}^{N}\right\}
$$

with $N=2^{m}-1$. Each failure mode $\mathcal{L}^{i},(i=1,2, \ldots, N)$ is therefore an element of the set $\mathcal{L}$. We also view $\mathcal{L}^{i}$ as a matrix, i.e., as a particular pattern of matrix $\mathcal{L}$ in (4) depending on the values of $l_{j}(j=1,2, \ldots m)$. Throughout, when $\mathcal{L}$ is invoked as a matrix, it will mean that matrix $\mathcal{L}$ varies over the set of matrices in (5). Note that the faulty mode $\mathcal{L}^{i}$ in the NCS architecture of figure 1 is estimated by the FDI unit. In order to ensure that system (1) should remain controllable, we assume that the set $\mathcal{L}$ excludes the element $\operatorname{diag}\{0,0, \ldots, 0\}$, i.e., at least one actuator should be healthy. We further assume that the time-varying delay $\tau(k)$ lies between the following positive integer bounds $\tau_{m}$ and $\tau_{M}$, i.e.,

$$
\tau_{m} \leq \tau(k) \leq \tau_{M}
$$

Given positive definite symmetric matrices $Q_{1}$ and $Q_{2}$, we consider the quadratic cost function:

$$
J=\sum_{k=0}^{\infty}\left[x^{T}(k) Q_{1} x(k)+u^{T}(k) Q_{2} u(k)\right]
$$

and with respect to this cost function, we define the guaranteed cost controller in the event of actuator failures as follows.

Definition 1: If there exists a control law $u(k)$ and a positive scalar $J^{*}$ such that, for all admissible uncertainties $\Delta(k)$ and all failure modes $\mathcal{L}^{i} \in \mathcal{L}$, the closed-loop system (1)-(3) is stable with cost function (7) satisfying $J \leq J^{*}$, then $J^{*}$ is said to be a guaranteed cost and $u(k)$ a guaranteed cost controller for the uncertain system (1).

In the next section, we will proceed through two main steps to design a cost guaranteed active fault tolerant control in the NCS framework. These steps are :

- (i) construct a fault-tolerant controller (i.e., a robust controller), with structure as given by (3), which achieves the smallest possible value for $J^{*}$ under all admissible plant uncertainties and all actuator failure modes

- (ii) redesign that part of the above controller associated to only one fault-free actuator in order to improve the robust performance without loss of the stability property of the design in step (i). Step (ii) repeats for all $m$ actuators and results in a bank of $m$ controllers.

It follows from inequality $m \leq N=2^{m}-1$, that the cardinality of the bank of controllers (which is equal to the number of actuators) is less than the cardinality of the set $\mathcal{L}$ of faulty modes. For each faulty mode $\mathcal{L}^{i}$, the controller to be switched-on should be the best as ranked with respect to a closed-loop performance index. In this paper, we will not address the switching and reconfiguration mechanism, we focus on the design of the bank of $m$ controllers

\section{AFTC DESIGN FOR NCS}

\section{A. Step (i): Robust Stability}

The control law (3) applied to plant (1) results in the following system:

$$
x(k+1)=A_{1} x(k)+B \mathcal{L} K x(k-\tau(k))
$$

where $A_{1}=A+D \Delta(k) E$. The cost function associated to system (8) is

$$
J=\sum_{k=0}^{\infty} x_{e}^{T}(k) Q x_{e}(k)
$$

where $x_{e}^{T}(k)=\left[x^{T}(k), \quad x^{T}(k-\tau(k))\right]$, and $Q=$ $\operatorname{diag}\left\{Q_{1}, K^{T} Q_{2} K\right\}$. Under the assumptions made in section II, we state the following result:

Theorem 1: If there exists a gain matrix $K$, a scalar $\epsilon>$ 0 , symmetric positive-definite matrices $P_{1} \in \mathcal{R}^{n \times n}, R \in$ $\mathcal{R}^{n \times n}, S \in \mathcal{R}^{n \times n}$, and matrices $P_{2} \in \mathcal{R}^{n \times n}, P_{3} \in \mathcal{R}^{n \times n}$, $W \in \mathcal{R}^{2 n \times 2 n}, M \in \mathcal{R}^{2 n \times n}$ such that the following matrix inequalities are satisfied:

$$
\begin{aligned}
& {\left[\begin{array}{ccc}
\Gamma & P^{T}\left[\begin{array}{c}
0 \\
B \mathcal{L} K
\end{array}\right]-M & {\left[\begin{array}{c}
E^{T} \\
0
\end{array}\right]} \\
* & -R+K^{T} Q_{2} K & 0 \\
* & * & -\epsilon I
\end{array}\right]<0} \\
& {\left[\begin{array}{cc}
W & M \\
* & S
\end{array}\right] \geq 0}
\end{aligned}
$$

with

$$
\begin{aligned}
& \Gamma=P^{T}\left[\begin{array}{cc}
0 & I \\
A-I & -I
\end{array}\right]+\left[\begin{array}{cc}
0 & I \\
A-I & -I
\end{array}\right]^{T} P \\
& +\epsilon P^{T}\left[\begin{array}{cc}
0 & 0 \\
0 & D D^{T}
\end{array}\right] P+\left[\begin{array}{cc}
\mu R+Q_{1} & 0 \\
0 & P_{1}+\tau_{M} S
\end{array}\right] \\
& +\tau_{M} W+\left[\begin{array}{ll}
M & 0
\end{array}\right]+\left[\begin{array}{ll}
M & 0
\end{array}\right]^{T} \\
& \mu=1+\left(\tau_{M}-\tau_{m}\right), \quad P=\left[\begin{array}{cc}
P_{1} & 0 \\
P_{2} & P_{3}
\end{array}\right]
\end{aligned}
$$

Then, system (8) is asymptotically stable and the cost function (9) satisfies the inequality:

$$
\begin{aligned}
J \leq & x^{T}(0) P_{1} x(0)+\sum_{l=-\tau_{M}}^{-1} x^{T}(l) R x(l) \\
& +\sum_{\theta=-\tau_{M}+1}^{0} \sum_{l=-1+\theta}^{-1} y^{T}(l) S y(l) \\
& +\sum_{\theta=-\tau_{M}+2}^{-\tau_{m}+1} \sum_{l=\theta-1}^{-1} x^{T}(l) R x(l)
\end{aligned}
$$


where $y(l)=x(l+1)-x(l)$.

Proof: See the appendix.

Remark 1: The $*$ represents blocks that are readily inferred by symmetry

Remark 2: Note that the upper bound in equation (12) depends on the initial condition of system (8). To remove the dependence on the initial condition, we suppose that the initial state of system (8) might be arbitrary but belongs to the set $\mathcal{S}=\left\{x(l) \in \mathcal{R}^{n}: x(l)=U v, v^{T} v \leq 1, l=\right.$ $\left.-\tau_{M},-\tau_{M}+1, \ldots,-\tau_{m}\right\}$, where $U$ is a given matrix. Then inequality (12) leads to:

$$
J \leq \lambda_{\max }\left(U^{T} P_{1} U\right)+\rho_{1} \lambda_{\max }\left(U^{T} R U\right)+\rho_{2} \lambda_{\max }\left(U^{T} S U\right)
$$

where $\lambda_{\max }(\cdot)$ denotes the maximum eigenvalue of matrix $(\cdot), \rho_{1}=\mu\left(\tau_{M}+\tau_{m}\right) / 2$ and $\rho_{2}=2 \tau_{M}\left(\tau_{M}+1\right)$.

\section{B. Step (i): Controller Design}

By Sherman-Morrison matrix inversion formula, we have:

$$
P^{-1}=\left[\begin{array}{cc}
P_{1}^{-1} & 0 \\
-P_{3}^{-1} P_{2} P_{1}^{-1} & P_{3}^{-1}
\end{array}\right]
$$

In the sequel, we will denote $X=P_{1}^{-1}, Y=P_{3}^{-1}$ and $Z=-P_{3}^{-1} P_{2} P_{1}^{-1}$. We further restrict $M$ to the following case in order to obtain a linear matrix inequality (LMI) (see e.g. [7]):

$$
M=\delta P^{T}\left[\begin{array}{c}
0 \\
B \mathcal{L} K
\end{array}\right]
$$

where $\delta$ is a scalar parameter. Pre- and post-multiply equation (10) by $\operatorname{diag}\left\{\left(P^{-1}\right)^{T}, P_{1}^{-1}, I\right\}$ and $\operatorname{diag}\left\{P^{-1}\right.$, $\left.P_{1}^{-1}, I\right\}$ respectively; also pre- and post-multiply equation (11) by $\operatorname{diag}\left\{\left(P^{-1}\right)^{T}, P_{1}^{-1}\right\}$ and $\operatorname{diag}\left\{P^{-1}, P_{1}^{-1}\right\}$ and denote:

$$
\begin{array}{r}
L=P_{1}^{-1} R P_{1}^{-1}, F=K P_{1}^{-1}, \bar{S}=S^{-1}, \\
\left(P^{-1}\right)^{T} W P^{-1}=\left[\begin{array}{cc}
\bar{W}_{1} & \bar{W}_{2} \\
* & \bar{W}_{3}
\end{array}\right] .
\end{array}
$$

Applying the Schur complement and expanding the block matrices, we obtain the following result under the assumptions made in section II.

Theorem 2: Suppose that for a prescribed scalar $\delta$, there exists a scalar $\epsilon>0$, matrices $X>0, Y, Z, F, L>$ $0, \bar{S}>0, \bar{W}_{1}, \bar{W}_{2}, \bar{W}_{3}$, such that the following matrix inequalities are satisfied:

$$
\begin{gathered}
{\left[\begin{array}{cccc}
\Psi_{1} & \Psi_{2} & 0 & \Psi_{41} \\
* & \Psi_{3} & (1-\delta) B \mathcal{L} F & \Psi_{42} \\
* & * & -L & \Psi_{43} \\
* & * & * & \Psi_{5}
\end{array}\right]<0} \\
\\
{\left[\begin{array}{ccc}
\bar{W}_{1} & \bar{W}_{2} & 0 \\
* & \bar{W}_{3} & \delta B \mathcal{L} F \\
* & * & X \bar{S}^{-1} X
\end{array}\right] \geq 0}
\end{gathered}
$$

where

$$
\begin{aligned}
& \Psi_{1}=Z+Z^{T}+\mu L+\tau_{M} \bar{W}_{1} \\
& \Psi_{2}=Y+X(A-I)^{T}-Z^{T}+\tau_{M} \bar{W}_{2}+\delta(B \mathcal{L} F)^{T} \\
& \Psi_{3}=-Y-Y^{T}+\tau_{M} \bar{W}_{3}+\epsilon D D^{T}
\end{aligned}
$$

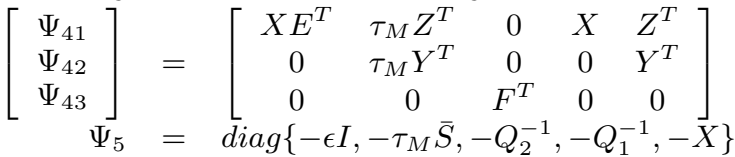

Then, the control law

$$
u(k)=F X^{-1} x(k-\tau(k))
$$

is a guaranteed cost networked control law for system (1) and the corresponding cost function satisfies:

$$
\begin{aligned}
J \leq & \lambda_{\max }\left(U^{T} X^{-1} U\right)+\rho_{1} \lambda_{\max }\left(U^{T} X^{-1} L X^{-1} U\right) \\
& +\rho_{2} \lambda_{\max }\left(U^{T} \bar{S}^{-1} U\right)
\end{aligned}
$$

where $\rho_{1}=\mu\left(\tau_{M}+\tau_{m}\right) / 2$ and $\rho_{2}=2 \tau_{M}\left(\tau_{M}+1\right)$.

Remark 3: From (17), we establish the following inequalities:

$$
\begin{gathered}
{\left[\begin{array}{cc}
-\alpha I & U^{T} \\
* & -X
\end{array}\right]<0,\left[\begin{array}{cc}
-\beta I & U^{T} \\
* & -X L^{-1} X
\end{array}\right]<0,} \\
{\left[\begin{array}{cc}
-\gamma I & U^{T} \\
* & -\bar{S}
\end{array}\right]<0}
\end{gathered}
$$

where $\alpha, \beta$, and $\gamma$ are scalars to be determined. It is worth noting that condition (18) is not a LMI because of the term $-X L^{-1} X$. This is also the case for condition (15) which is not a LMI because of the term $X \bar{S}^{-1} X$. Note that for any matrix $X>0$, we have:

$$
X \bar{S}^{-1} X \geq 2 X-\bar{S}, \quad X L^{-1} X \geq 2 X-L
$$

Given a prescribed scalar $\delta$, the design problem of the optimal guaranteed cost controller can be formulated therefore as the following optimization problem:

$$
\begin{aligned}
& \text { OP1: } \min _{\epsilon, X, Y, Z, F, L, \bar{S}, \bar{W}_{1}, \bar{W}_{2}, \bar{W}_{3}}\left(\alpha+\rho_{1} \beta+\rho_{2} \gamma\right)
\end{aligned}
$$

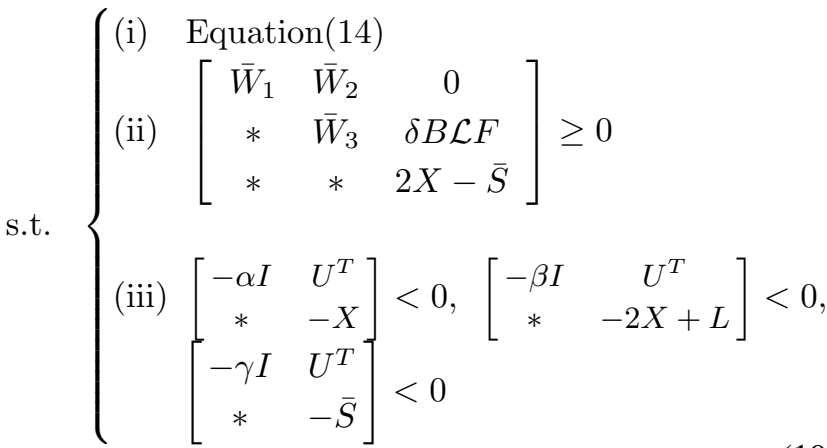

Clearly, the above optimization problem (19) is a convex optimization problem which can be effectively solved by existing LMI software [8]. Thus, the minimization of $\alpha+$ $\rho_{1} \beta+\rho_{2} \gamma$ implies the minimization of the cost in (9). By applying a simple one-dimensional search over $\delta$ for a certain $\tau_{M}$, a global optimum cost can be found. 


\section{Step (ii): Robust Stability}

Based on the controller designed in Theorem 2, let us assume that actuator $i$ is fault-free, then we can redesign the $i$-th row of controller gain matrix $K$ to improve the robust performance for the system against actuator failures. We can rewrite the overall control system as

$$
x(k+1)=A_{1} x(k)+\left(B_{\bar{i}} \mathcal{L}_{\bar{i}} K_{\bar{i}}+b_{i} k_{i}\right) x(k-\tau(k))
$$

where $A_{1}=A+D \Delta(k) E$, matrix $K_{\bar{i}}$ is obtained by deleting the $i$-th row from $K, B_{\bar{i}}$ is obtained by deleting the $i$-th column from $B$ and $\mathcal{L}_{\bar{i}}$ is obtained by deleting $i$-th row and $i$-th column from $\mathcal{L}$. The cost function associated to system (20) reads as:

$$
J=\sum_{k=0}^{\infty} x_{e}^{T}(k) Q x_{e}(k)
$$

with $x_{e}^{T}(k)=\left[x^{T}(k), \quad x^{T}(k-\tau(k))\right], \quad Q=$ $\operatorname{diag}\left\{Q_{1}, k_{i}^{T} Q_{2 i} k_{i}+K_{\bar{i}}^{T} Q_{2 \bar{i}} K_{\bar{i}}\right\}$, where $Q_{2 \bar{i}}$ is obtained by deleting the $i$-th row and $i$-th column from $Q_{2}$. With regard to system (20) where $K_{\bar{i}}$ is assumed to be known, we have the following result

Theorem 3: If there exists a gain matrix $k_{i}$, a scalar $\epsilon>$ 0 , symmetric positive-definite matrices $P_{1} \in \mathcal{R}^{n \times n}, R \in$ $\mathcal{R}^{n \times n}, S \in \mathcal{R}^{n \times n}$, and matrices $P_{2} \in \mathcal{R}^{n \times n}, P_{3} \in \mathcal{R}^{n \times n}$, $W \in \mathcal{R}^{2 n \times 2 n}, M \in \mathcal{R}^{2 n \times n}$ such that the following matrix inequalities are satisfied:

$$
\begin{aligned}
{\left[\begin{array}{ccc}
\Gamma & P^{T}\left[\begin{array}{c}
0 \\
B_{\bar{i}} \mathcal{L}_{\bar{i}} K_{\bar{i}}+b_{i} k_{i}
\end{array}\right]-M & {\left[\begin{array}{c}
E^{T} \\
0
\end{array}\right]} \\
* & -R+k_{i}^{T} Q_{2 i} k_{i}+K_{\bar{i}}^{T} Q_{2 \bar{i}} K_{\bar{i}} & 0 \\
* & * & -\epsilon I
\end{array}\right] } & <0, \\
{\left[\begin{array}{cc}
W & M \\
* & S
\end{array}\right] } & \geq 0 .
\end{aligned}
$$

Then, system (20) is asymptotically stable and the cost function (21) satisfies inequality (12).

Proof: Similar to the proof of Theorem 1.

\section{Step (ii): Controller Redesign}

Proceeding as in Step (i), we restrict $M$ to the following case in order to obtain a LMI:

$$
M=\delta P^{T}\left[\begin{array}{c}
0 \\
b_{i} k_{i}
\end{array}\right]
$$

where $\delta$ is a scalar parameter. Pre- and post-multiply equation (22) with $\operatorname{diag}\left\{\left(P^{-1}\right)^{T}, P_{1}^{-1}, I\right\}$ and $\operatorname{diag}\left\{P^{-1}\right.$, $\left.P_{1}^{-1}, I\right\}$ respectively; also pre- and post-multiply equation (23) with $\operatorname{diag}\left\{\left(P^{-1}\right)^{T}, P_{1}^{-1}\right\}$ and $\operatorname{diag}\left\{P^{-1}, P_{1}^{-1}\right\}$ respectively and denote:

$$
\begin{array}{r}
L=P_{1}^{-1} R P_{1}^{-1}, F^{*}=k_{i} P_{1}^{-1}, \bar{S}=S^{-1}, \\
\left(P^{-1}\right)^{T} W P^{-1}=\left[\begin{array}{cc}
\bar{W}_{1} & \bar{W}_{2} \\
* & \bar{W}_{3}
\end{array}\right] .
\end{array}
$$

The Schur complement trick leads to the following controller redesign result.

Theorem 4: Suppose that for a prescribed scalar $\delta$, there exists a scalar $\epsilon>0$, matrices $X>0, Y, Z, F^{*}, L>$
$0, \bar{S}>0, \bar{W}_{1}, \bar{W}_{2}, \bar{W}_{3}$, such that the following matrix inequalities are satisfied:

$$
\begin{aligned}
& {\left[\begin{array}{cccc}
\tilde{\Psi}_{1} & \tilde{\Psi}_{2} & 0 & \tilde{\Psi}_{41} \\
* & \tilde{\Psi}_{3} & B_{\bar{i}} \mathcal{L}_{\bar{i}} K_{\bar{i}} X+(1-\delta) b_{i} F^{*} & \tilde{\Psi}_{42} \\
* & * & -L & \tilde{\Psi}_{43} \\
* & * & * & \tilde{\Psi}_{5}
\end{array}\right]<0} \\
& {\left[\begin{array}{ccc}
\bar{W}_{1} & \bar{W}_{2} & 0 \\
* & \bar{W}_{3} & \delta b_{i} F^{*} \\
* & * & X \bar{S}^{-1} X
\end{array}\right] \geq 0}
\end{aligned}
$$

where

$$
\begin{aligned}
& \tilde{\Psi}_{1}=Z+Z^{T}+\mu L+\tau_{M} \bar{W}_{1} \\
& \tilde{\Psi}_{2}=Y+X(A-I)^{T}-Z^{T}+\tau_{M} \bar{W}_{2}+\delta\left(b_{i} F^{*}\right)^{T} \\
& \tilde{\Psi}_{3}=-Y-Y^{T}+\tau_{M} \bar{W}_{3}+\epsilon D D^{T} \\
& \begin{aligned}
{\left[\begin{array}{c}
\tilde{\Psi}_{41} \\
\tilde{\Psi}_{42} \\
\tilde{\Psi}_{43}
\end{array}\right] } & =\left[\begin{array}{cccccc}
X E^{T} & \tau_{M} Z^{T} & 0 & 0 & X & Z^{T} \\
0 & \tau_{M} Y^{T} & 0 & 0 & 0 & Y^{T} \\
0 & 0 & \left(F^{*}\right)^{T} & X K_{\bar{i}}^{T} & 0 & 0
\end{array}\right] \\
\tilde{\Psi}_{5} & =\operatorname{diag}\left\{-\epsilon I,-\tau_{M} \bar{S},-Q_{2 i}^{-1},-Q_{2 \bar{i}}^{-1},-Q_{1}^{-1},-X\right\}
\end{aligned}
\end{aligned}
$$

Then, the $i$ th control law

$$
u_{i}(k)=F^{*} X^{-1} x(k-\tau(k))
$$

is a guaranteed cost networked control law of system (20) and the corresponding cost function satisfies:

$$
\begin{aligned}
J \leq & \lambda_{\max }\left(U^{T} X^{-1} U\right)+\rho_{1} \lambda_{\max }\left(U^{T} X^{-1} L X^{-1} U\right) \\
& +\rho_{2} \lambda_{\max }\left(U^{T} \bar{S}^{-1} U\right)
\end{aligned}
$$

where $\rho_{1}=\mu\left(\tau_{M}+\tau_{m}\right) / 2$ and $\rho_{2}=2 \tau_{M}\left(\tau_{M}+1\right)$.

Given a prescribed scalar $\delta$, the redesign problem of the optimal guaranteed cost controller can be formulated as the following convex optimization problem:

$$
\begin{aligned}
& \text { OP2: } \min _{\epsilon, X, Y, Z, F^{*}, L, \bar{S}, \bar{W}_{1}, \bar{W}_{2}, \bar{W}_{3}}\left(\alpha+\rho_{1} \beta+\rho_{2} \gamma\right)
\end{aligned}
$$

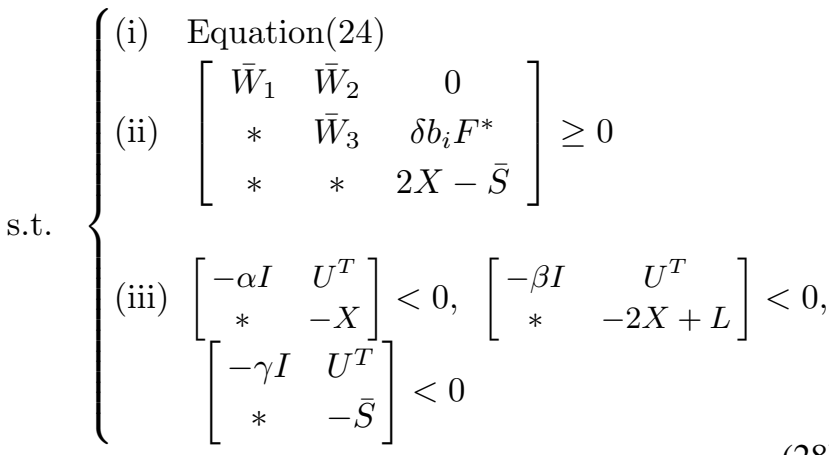

\section{ILLuStrative EXAMPLE}

The dynamics are described as follows:

$$
\begin{aligned}
& A=\left[\begin{array}{cc}
0.9 & 0 \\
0.2 & 0.5
\end{array}\right], B=\left[\begin{array}{cc}
0.2 & 0.1 \\
0 & -0.1
\end{array}\right], \\
& D=\left[\begin{array}{cc}
0 & 0.1 \\
0.1 & 0
\end{array}\right], E=\left[\begin{array}{cc}
0.1 & 0 \\
0.1 & -0.1
\end{array}\right],
\end{aligned}
$$

and the simulation parameters are given as:

$$
Q_{1}=\left[\begin{array}{ll}
1 & 0 \\
0 & 1
\end{array}\right], \quad Q_{2}=\left[\begin{array}{cc}
0.1 & 0 \\
0 & 0.1
\end{array}\right], \quad U=\left[\begin{array}{ll}
1 & 0 \\
0 & 1
\end{array}\right]
$$


When $\tau_{m}=1, \tau_{M}=2$ and $\delta=1$, by OP1 (19), the cost is obtained as $J_{1}=61.6653$ and the fault-tolerant controller can be designed for Step (i):

$$
\left[\begin{array}{l}
k_{1} \\
k_{2}
\end{array}\right]=\left[\begin{array}{ll}
-0.0812 \times 10^{-5} & -0.1333 \times 10^{-5} \\
-0.1865 \times 10^{-5} & -0.3060 \times 10^{-5}
\end{array}\right] .
$$

In Step (ii), by OP2 (28), the cost and the feedback gains are redesigned as

$$
\begin{aligned}
& J_{2}=39.0026, k_{1}^{*}=\left[\begin{array}{ll}
-0.8776 & -0.2857
\end{array}\right], \\
& J_{3}=49.9616, k_{2}^{*}=\left[\begin{array}{ll}
-0.6494 & -0.4161
\end{array}\right] .
\end{aligned}
$$

As a result, the two controllers are determined as follows:

$$
\begin{aligned}
& \sharp 1:\left[\begin{array}{l}
k_{1}^{*} \\
k_{2}
\end{array}\right]=\left[\begin{array}{cc}
-0.8776 & -0.2857 \\
-0.1865 \times 10^{-5} & -0.3060 \times 10^{-5}
\end{array}\right], \\
& \sharp 2:\left[\begin{array}{l}
k_{1} \\
k_{2}^{*}
\end{array}\right]=\left[\begin{array}{cc}
-0.0812 \times 10^{-5} & -0.1333 \times 10^{-5} \\
-0.6494 & -0.4161
\end{array}\right] .
\end{aligned}
$$

In the simulation, the step disturbance 1 as shown in Fig. 2-(a) enters into the system at time instant 35 and disappears at time instant 40 . The step disturbance 2 as shown in Fig. 2-(b) enters into the system at time instant 5 and disappears at time instant 10 . In figure 2-(c), the solid line represents the failure of actuator 1 which occurs at time instant 15 and disappears at time instant 35, occurs again at time instant 55 and disappears at time instant 65 . In figure 2-(d), the solid line represents the failure of actuator 2 which occurs at time instant 35 and disappears at time instant 45 , occurs again at time instant 65 and disappears at time instant 80 . The delay of fault detection is assumed to be 3 time steps, which is represented by dash-dotted lines as shown in figure 2 (c) and (d). Under the above simulation setting, the state responses are shown in figures 2 (e) and (f):

- the dotted line represents the state response for controller-switching sequence $\mathrm{N}^{\circ} 1$ : $\sharp 2$ is the initial controller, and $\sharp 1$ is switched-on at time instant 38 , then $\sharp 2$ at time instant $48, \sharp 1$ at time instant 68 ;

- the solid line represents the state response for controllerswitching sequence $\mathrm{N}^{\circ} 2: \sharp 1$ is the initial controller, and $\sharp 2$ is switched-on at time instant 38 , then $\sharp 1$ at time instant $48, \sharp 2$ at time instant 68 ;

- the dashed line represents the state response under the fault tolerant control of step (i);

- the dot-dashed line represents state response under standard discrete-time linear quadratic regulator design without considering any time-delay and faults, which is constructed by the Matlab command dlqr.

The trace of matrices $\frac{\left(x^{*}\right)\left(x^{*}\right)^{T}}{\text { simulation step }}$ is used as a performance measure for comparison, where $x^{*}$ represents the state trajectory in the different schemes. After computation, we obtain for the above four scenarios the traces $T r_{1}=$ $0.0279, \operatorname{Tr}_{2}=0.0338, T r_{3}=0.0499, \operatorname{Tr}_{4}=0.4236$ which means that $T r_{1}<T r_{2}<T r_{3}<T r_{4}$. We can draw the conclusion that the proposed method for sequence $\mathrm{N}^{\circ} 1$ is the best control scheme, and in all possible switching scenarios with controllers in the designed bank, the proposed active FTC is able to guarantee at least the closed-loop stability of the overall system.

\section{CONCLUSion}

In this paper, the stability guaranteed active fault tolerant control against actuators failure in networked control system with time-varying but bounded delays has been addressed. A detailed design procedure is formulated as a convex optimization problem which can be efficiently solved by existing software. An illustrative example is also given to show the efficiency of the proposed method for networked control systems.

\section{APPENDIX:PROOF OF THEOREM 1}

The following matrix inequalities are essential for the proof of theorem 1:

Lemma 1: [9] Assume that $a(\cdot) \in \mathcal{R}^{n_{a}}, b(\cdot) \in \mathcal{R}^{n_{b}}$, and $N(\cdot) \in \mathcal{R}^{n_{a} \times n_{b}}$. Then, for any matrices $X \in \mathcal{R}^{n_{a} \times n_{a}}, Y \in$ $\mathcal{R}^{n_{a} \times n_{b}}$, and $Z \in \mathcal{R}^{n_{b} \times n_{b}}$, the following holds:

$$
-2 a^{T} N b \leq\left[\begin{array}{c}
a \\
b
\end{array}\right]^{T}\left[\begin{array}{cc}
X & Y-N \\
Y^{T}-N^{T} & Z
\end{array}\right]\left[\begin{array}{l}
a \\
b
\end{array}\right]
$$

where $\left[\begin{array}{cc}X & Y \\ Y^{T} & Z\end{array}\right] \geq 0$.

Lemma 2: [10] Let $Y$ be a symmetric matrix and $H, E$ be given matrices with appropriate dimensions, then

$$
Y+H F E+E^{T} F^{T} H^{T}<0
$$

holds for all $F$ satisfying $F^{T} F \leq I$, if and only if there exists a scalar $\epsilon>0$ such that:

$$
Y+\epsilon H H^{T}+\epsilon^{-1} E^{T} E<0
$$

Proof: Note that $x(k-\tau(k))=x(k)-\sum_{l=k-\tau(k)}^{k-1} y(l)$, where $y(l)=x(l+1)-x(l)$. Then from system (8), we have:

$$
0=\left(A_{1}+B \mathcal{L} K-I\right) x(k)-y(k)-B \mathcal{L} K \sum_{l=k-\tau(k)}^{k-1} y(l)
$$

Choose the Lyapunov-Krasovskii function candidates as follows:

$$
V(k)=V_{1}(k)+V_{2}(k)+V_{3}(k)
$$

where

$$
\begin{aligned}
V_{1}(k) & =x^{T}(k) P_{1} x(k) \\
V_{2}(k) & =\sum_{l=k-\tau(k)}^{k-1} x^{T}(l) R x(l) \\
V_{3}(k) & =\sum_{\theta=-\tau_{M}}^{-1} \sum_{l=k+\theta}^{k-1} y^{T}(l) S y(l) \\
& +\sum_{\theta=-\tau_{M}+2}^{-\tau_{m}+1} \sum_{l=k+\theta-1}^{k-1} x^{T}(l) R x(l)
\end{aligned}
$$

Taking the forward difference for the Lyapunov functional $V_{1}(k)$, we have: 
(a)

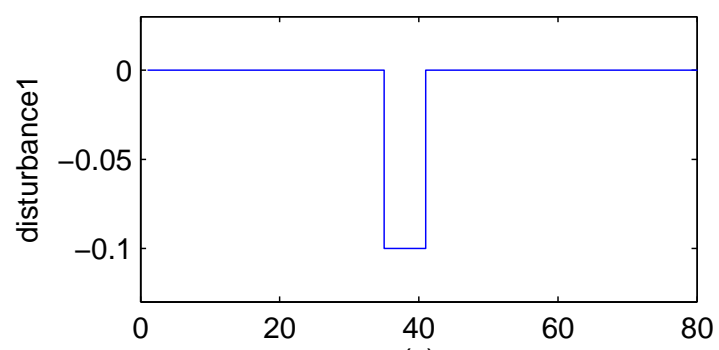

(c)

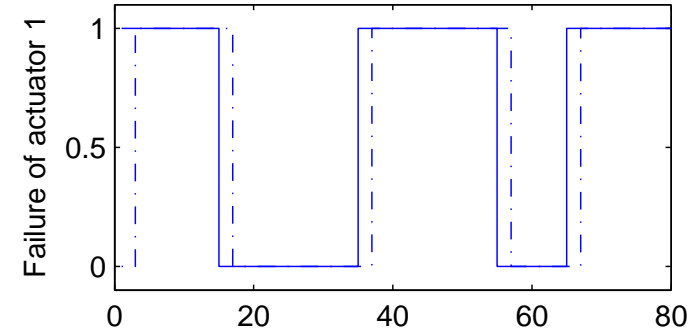

(e)

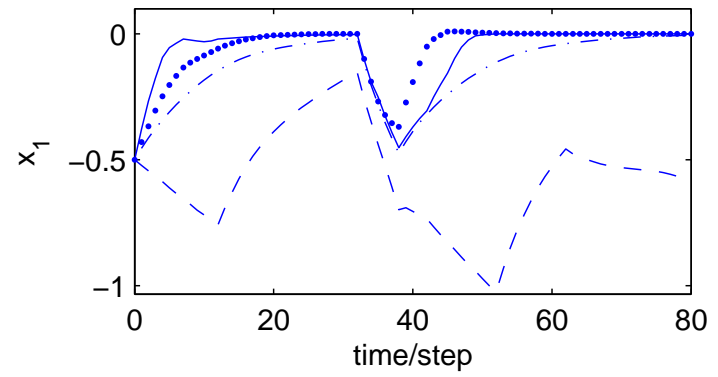

(b)

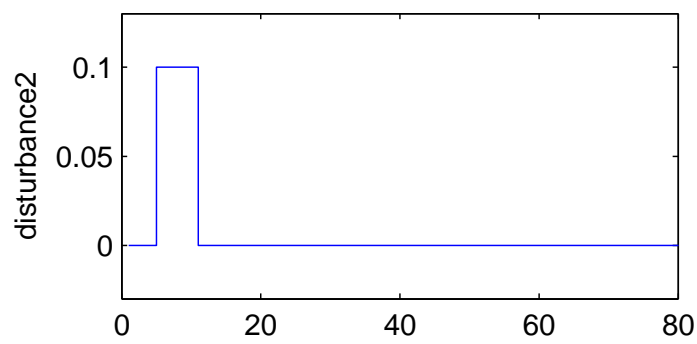

(d)
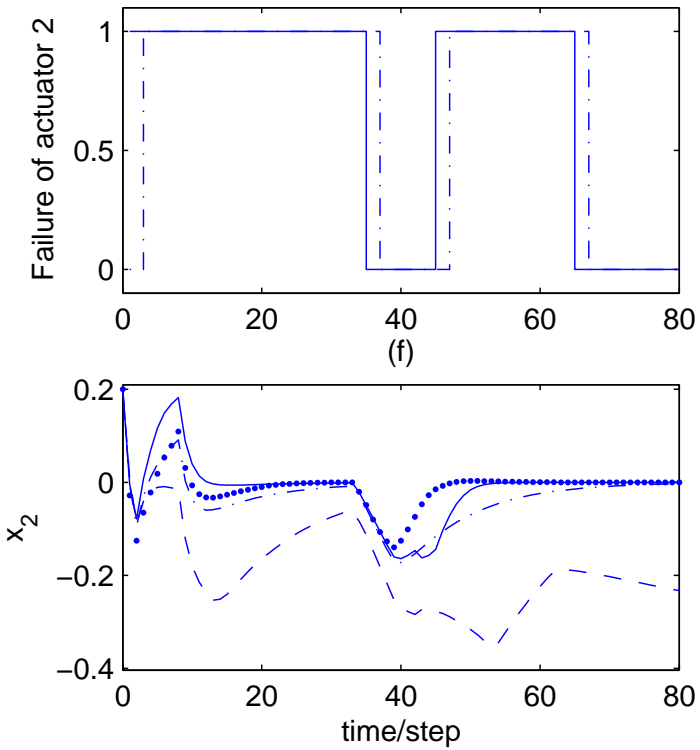

Fig. 2. Disturbance, Actuator Failures and State Response

$$
\Delta V_{1}(k)=2 x^{T}(k) P_{1} y(k)+y^{T}(k) P_{1} y(k)
$$

From (29), we have:

$$
\begin{aligned}
& 2 x^{T}(k) P_{1} y(k) \\
& =2 \eta^{T}(k) P^{T}\left[\left(A_{1}+B \mathcal{L} K-I\right) x(k)-y(k)-B \mathcal{L} K \sum_{l=k-\tau(k)}^{k-1} y(l)\right]
\end{aligned}
$$

where $\eta^{T}(k)=\left[\begin{array}{ll}x^{T}(k) & y^{T}(k)\end{array}\right]$. Choose constant matrices $W, M$ and $S$ satisfying (11), by Lemma 1, we have:

$$
\begin{aligned}
-2 & \sum_{l=k-\tau(k)}^{k-1} \eta^{T}(k) P^{T}\left[\begin{array}{c}
0 \\
B \mathcal{L} K
\end{array}\right] y(l) \\
\leq & \tau_{M} \eta^{T}(k) W \eta(k)+\sum_{l=k-\tau_{M}}^{k-1} y^{T}(l) S y(l) \\
& +2 \eta^{T}(k)\left\{M-P^{T}\left[\begin{array}{c}
0 \\
B \mathcal{L} K
\end{array}\right]\right\}(x(k)-x(k-\tau(k))
\end{aligned}
$$

Similarly,

$$
\begin{aligned}
\Delta V_{2}(k) & =x^{T}(k) R x(k)-x^{T}(k-\tau(k)) R x(k-\tau(k)) \\
& +\sum_{l=k+1-\tau(k+1)}^{k-1} x^{T}(l) R x(l)-\sum_{k-\tau(k)+1}^{k-1} x^{T}(l) R x(l)
\end{aligned}
$$

Note that:

$$
\begin{aligned}
& \sum_{l=k+1-\tau(k+1)}^{k-1} x^{T}(l) R x(l) \\
= & \sum_{l=k+1-\tau_{m}}^{k-1} x^{T}(l) R x(l)+\sum_{l=k+1-\tau(k+1)}^{k-\tau_{m}} x^{T}(l) R x(l) \\
\leq & \sum_{l=k+1-\tau(k)}^{k-1} x^{T}(l) R x(l)+\sum_{l=k+1-\tau_{M}}^{k-\tau_{m}} x^{T}(l) R x(l)
\end{aligned}
$$

So, we have:

$$
\begin{array}{rl}
\Delta V_{2}(k) \leq x^{T}(k) R & x(k)-x^{T}(k-\tau(k)) R x(k-\tau(k)) \\
& +\sum_{l=k+1-\tau_{M}}^{k-\tau_{m}} x^{T}(l) R x(l)
\end{array}
$$

Furthermore, we have:

$$
\Delta V_{3}(k)=\tau_{M} y^{T}(k) S y(k)-\sum_{l=k-\tau_{M}}^{k-1} y^{T}(l) S y(l)
$$




$$
+\left(\tau_{M}-\tau_{m}\right) x^{T}(k) R x(k)-\sum_{l=k+1-\tau_{M}}^{k-\tau_{m}} x^{T}(l) R x(l)
$$

Combining (9) and (31)-(35), we have:

$$
\begin{aligned}
\Delta V(k) & \leq \xi^{T}(k)\left[\Theta_{0}\left(\tau_{m}, \tau_{M}\right)+\hat{D} \Delta(k) \hat{E}+\hat{E}^{T} \Delta^{T}(k) \hat{D}^{T}\right] \xi(k) \\
& -x_{e}^{T}(k) Q x_{e}(k)
\end{aligned}
$$

where

$$
\begin{aligned}
& \xi^{T}(k)=\left[\begin{array}{ll}
\eta^{T}(k) & x^{T}(k-\tau(k))
\end{array}\right], \\
& \left.\hat{D}^{T}=\left[\left[\begin{array}{ll}
0 & D^{T}
\end{array}\right] P \quad 0\right], \hat{E}=\left[\begin{array}{ll}
E & 0
\end{array}\right] 0\right] \text {, } \\
& \Theta_{0}\left(\tau_{m}, \tau_{M}\right)=\left[\begin{array}{cc}
\Gamma_{0} & P^{T}\left[\begin{array}{c}
0 \\
B \mathcal{L} K
\end{array}\right]-M \\
* & -R+K^{T} Q_{2} K
\end{array}\right], \\
& \Gamma_{0}=\Gamma-\epsilon P^{T}\left[\begin{array}{cc}
0 & 0 \\
0 & D D^{T}
\end{array}\right] P
\end{aligned}
$$

By Lemma 2, we have:

$$
\begin{aligned}
\Delta V(k) & \leq \xi^{T}(k)\left[\Theta_{0}\left(\tau_{m}, \tau_{M}\right)+\epsilon \hat{D} \hat{D}^{T}+\epsilon^{-1} \hat{E}^{T} \hat{E}\right] \xi(k) \\
& -x_{e}^{T}(k) Q x_{e}(k)
\end{aligned}
$$

By Schur complement and from (10), we have:

$$
\Delta V(k) \leq x_{e}^{T}(k) Q x_{e}(k)
$$

Summing both sides of the above inequality from 0 to $\infty$ and using system stability yields equation (12), and from definition 1 , this completes the proof of the theorem.

\section{REFERENCES}

[1] M. Mariton, "Detection delays, false alarm rates and the reconfiguration of control systems," International Journal of Control, vol. 49, pp. 981-992, 1989

[2] M. Mahmoud, J. Jiang, and Y. Zhang, "Stabilization of active fault tolerant control systems with imperfect fault detection and diagnosis," Stochastic Analysis and Applications, vol. 21, no. 3, pp. 673-701, 2003.

[3] N. E. Wu, "Robust feedback design with optimized diagnostic performance," IEEE Trans. on Automatic Control, vol. 42, no. 9, pp $1264-1268,1997$.

[4] M. Maki, J. Jiang, and K. Hagino, "A stability guaranteed active fault-tolerant control against actuator failures," International Journal of Robust and Nonlinear Control, vol. 14, pp. 1061-1077, 2004.

[5] W. Zhang, M. S. Branicky, and S.M. Phillips, "Stability of networked control systems", IEEE Control Systems Magazine, vol. 21, no. 1, pp. 8499, 2001.

[6] Y. Tipsuwan, M-Y. Chow, "Control methodologies in networked control systems", Control Engineerong Practice, vol. 11, pp. 10991111,2003

[7] W. H. Chen, Z. H. Guan, and X. Lu, "Delay-dependent guaranteed cost control for uncertain discrete-time systems with delay," IEE Proc.Control Theory Appl., vol. 150, no. 4, pp. 412-416, July 2003.

[8] P. Gahinet, A. Nemirovski, A. J. Laub, and M. Chilali, LMI Control Toolbox-for Use with Matlab. The Math Works Inc., 1995.

[9] Y. S. Moon, P. Park, W. H. Kwon, and Y. S. Lee, "Delay-dependent robust stabilization of uncertain state-delayed systems," Int. J. Control, vol. 74, no. 14, pp. 1447-1455, 2001.

[10] Y. Wang, L. Xie, and C. E. D. Souza, "Robust control of a class of uncertain nonlinear systems," Systems and Control Letters, vol. 19, no. 3, pp. 139-149, 1992. 